\title{
New Fourth and Fifth-Order Iterative Methods for Solving Nonlinear Equations
}

\author{
Muhammad Saqib1, Muhammad Iqbal', Shahid Ali' ${ }^{1}$, Tariq Ismaeel2,3 \\ ${ }^{1}$ Department of Mathematics, Lahore Leads University, Lahore, Pakistan \\ ${ }^{2}$ Department of Mathematics, GC University, Lahore, Pakistan \\ ${ }^{3}$ School of Mathematics and Statistics, Xi'an Jiaotong University, Xi'an, China \\ Email: msaqib0321@hotmail.com, iqbal66dn@yahoo.com, Shahidali.2029@gmail.com, \\ tariqismaeel@gcu.edu.pk
}

Received 3 June 2015; accepted 10 July 2015; published 14 July 2015

Copyright (C) 2015 by authors and Scientific Research Publishing Inc.

This work is licensed under the Creative Commons Attribution International License (CC BY).

http://creativecommons.org/licenses/by/4.0/

(c) () Open Access

\begin{abstract}
In this paper, we establish two new iterative methods of order four and five by using modified homotopy perturbation technique. We also present the convergence analysis of these iterative methods. To assess the validity and performance of these iterative methods, we have applied to solve some nonlinear problems.
\end{abstract}

\section{Keywords}

Iterative Methods, Homotopy Perturbation Technique, Order of Convergence, Nonlinear Equations

\section{Introduction}

Consider the single variable nonlinear equation

$$
f(x)=0 .
$$

Finding the zeros (1) is an interesting and very ancient problem in numerical analysis. Newton and fixed point iterative methods are very old methods for solving nonlinear equations. Newton method is quadratically convergent where as fixed point method is linear convergent. Many modifications have been made in Newton's method to get cubically convergent iterative methods. Many higher order iterative methods have been established to approximate the solution of (1) by using different techniques including Taylor's series, quadrature rules, Adomain decomposition, homotopy perturbation, Gejji and Jafari decomposition, Noor decomposition, see the refrences [1]-[8]. Initialty, we do not put any restrictions on the original function $f$. In fixed point method, we rewrite $f(x)=0$ as $x=g(x)$ where 

(i) there exist $[a, b]$ such that $g(x) \in[a, b]$ for all $x \in[a, b]$,
(ii) there exist $[a, b]$ such that $|g(x)| \leq \lambda<1$ for all $x \in[a, b]$.

We shall establish fourth and fifth order iterative methods using modified homotopy perturbation technique. The order of convergence of a sequence of approximation is defined as;

Definition 1 [9] Let the sequence $\left\{x_{n}\right\}$ converges to $\alpha$. If there is a positive integer $p$ and real number $C$ such that

$$
\lim _{n \rightarrow \infty}\left|\frac{x_{n+1}-\alpha}{\left(x_{n}-\alpha\right)^{p}}\right|=C
$$

Then $p$ is order of convergence.

Theorem 1 (see [6]). Suppose that $\varphi \in C^{p}[a, b]$. If $\varphi^{(k)}(x)=0$, for $k=0,1,2, \cdots, m-1$ and $\varphi^{(p)}(x) \neq 0$, then the sequence $\left\{x_{n}\right\}$ is of order $m$.

\section{Development of New Methods}

Consider the nonlinear equation

$$
f(x)=0 .
$$

We can rewrite the above equation as

$$
x=g(x) .
$$

We suppose that $\alpha$ is a root of (2) and $\gamma$ is initial guess close to $\alpha$. We can rewrite (3) by using Taylor's expansion as:

$$
x=g(\gamma)+(x-\gamma) g^{\prime}(\gamma)+G(x)
$$

where

$$
G(x)=x-g(\gamma)+(x-\gamma) g^{\prime}(\gamma)
$$

We can rewrite (4) as

$$
x=\frac{g(\gamma)-\gamma g^{\prime}(\gamma)}{1-g^{\prime}(\gamma)}+\frac{G(x)}{1-g^{\prime}(\gamma)}
$$

It can be written in the form

$$
x=c+N(x)
$$

where

$$
c=\frac{g(\gamma)-\gamma g^{\prime}(\gamma)}{1-g^{\prime}(\gamma)}
$$

and

$$
N(x)=\frac{G(x)}{1-g^{\prime}(\gamma)}
$$

From (5), we see that

$$
G\left(x_{0}\right)=g\left(x_{0}\right)-x_{0} .
$$

We shall decompose the nonlinear operator $N(x)$ by using modified homotopy perturbation technique. For this, we construct a homotopy $H(x, p, m):(\mathbb{R} \times[0,1]) \times \mathbb{R} \rightarrow \mathbb{R}$, that satisfies

$$
H(x, p, m)=x-c-p N(x)-p(1-p) m=0 . \quad m, x \in \mathbb{R}, p \in(0,1]
$$


where $p$ is embedding parameter and $m$ is unknown real number. The embedding parameter $p$ is monotonically increases from zero to unity as the trivial problem

$$
H(x, 0, m)=x-c=0
$$

is continuously deformed the original problem

$$
H(x, 1, m)=x-c-N(x)=0 .
$$

The basic assumption of modified HPM is that the solution $x$ of (10) can be expressed as a power series in $p$ in the following form

$$
x=x_{0}+p^{1} x_{1}+p^{2} x_{2}+\cdots
$$

The approximate solution of (2) can be obtained as

$$
x=\operatorname{Lim}_{p \rightarrow 1} v=x_{0}+x_{1}+x_{2}+x_{3}+\cdots .
$$

The convergence of the infinite series (13) has been proved by He [10]. For the application of modified HPM to (2), we can rewrite (10) by expanding $N(x)$ into Taylor's expansion around $x_{0}$ :

$$
x-c-p\left\{N\left(x_{0}\right)+\left(x-x_{0}\right) N^{\prime}\left(x_{0}\right)+\left(x-x_{0}\right)^{2} N^{\prime \prime}\left(x_{0}\right)+\cdots\right\}-p(1-p) m=0
$$

By substituting (13) in (15), we have

$$
x_{0}+p^{1} x_{1}+p^{2} x_{2}+\cdots-c-p\left\{N\left(x_{0}\right)+\left(x-x_{0}\right) N^{\prime}\left(x_{0}\right)+\left(x-x_{0}\right)^{2} N^{\prime \prime}\left(x_{0}\right)+\cdots\right\}-p(1-p) m=0 .
$$

By equating the coefficients of like powers of $p$, we have

$$
\begin{gathered}
p^{0}: x_{0}-c=0 \\
p^{1}: x_{1}-N\left(x_{0}\right)-m=0 \\
p^{2}: x_{2}-x_{1} N^{\prime}\left(x_{0}\right)+m=0 \\
p^{3}: x_{3}-x_{2} N^{\prime}\left(x_{0}\right)-\frac{1}{2} x_{1}^{2} N^{\prime \prime}\left(x_{0}\right)=0 .
\end{gathered}
$$

We find the value of unknown parameter $m$ such that

$$
x_{2}=0 \text {. }
$$

From (17), we have

$$
x_{1}=N\left(x_{0}\right)+m .
$$

By putting value of $x_{1}$ and $x_{2}$ in (18) yields

$$
m=\frac{N\left(x_{0}\right) N^{\prime}\left(x_{0}\right)}{1-N^{\prime}\left(x_{0}\right)} .
$$

Substitution of (20) in (17) yields

$$
x_{1}=\frac{N\left(x_{0}\right)}{1-N^{\prime}\left(x_{0}\right)} .
$$

From (19), we get

$$
x_{3}=\frac{1}{2}\left(\frac{N\left(x_{0}\right)}{1-N^{\prime}\left(x_{0}\right)}\right)^{2} N^{\prime \prime}\left(x_{0}\right) .
$$

From (16), we have

$$
x_{0}=c \text {, }
$$


when

$$
x \approx x_{0}=c=\frac{g(\gamma)-\gamma g^{\prime}(\gamma)}{1-g^{\prime}(\gamma)} .
$$

This formulation allows us to form the following iterative method.

Algorithm 2 For any initial value $x_{0}$, we compute the approximation solution $x_{n+1}$, by the iterative method.

$$
x_{n+1}=\frac{g\left(x_{n}\right)-x_{n} g^{\prime}\left(x_{n}\right)}{1-g^{\prime}\left(x_{n}\right)}
$$

which is mainly due to Shin et al. [9] and has quadratic convergence.

When

$$
x \approx x_{0}+x_{1}=x_{0}+\frac{N\left(x_{0}\right)}{1-N^{\prime}\left(x_{0}\right)}=x_{0}-\frac{g\left(x_{0}\right)-x_{0}}{1-g^{\prime}\left(x_{0}\right)}=\frac{g\left(x_{0}\right)-x_{0} g^{\prime}\left(x_{0}\right)}{1-g^{\prime}\left(x_{0}\right)} .
$$

From this formulation, we suggest the following iterative method.

Algorithm 3 For any initial value $x_{0}$, we compute the approximation solution $x_{n+1}$, by the iterative method. Predictor step:

$$
y_{n}=\frac{g\left(x_{n}\right)-x_{n} g^{\prime}\left(x_{n}\right)}{1-g^{\prime}\left(x_{n}\right)} .
$$

Corrector step:

$$
x_{n+1}=\frac{g\left(y_{n}\right)-y_{n} g^{\prime}\left(y_{n}\right)}{1-g^{\prime}\left(y_{n}\right)} .
$$

When

$$
\begin{aligned}
& x \approx x_{0}+x_{1}+x_{2}+x_{3} \\
& =x_{0}+\frac{N\left(x_{0}\right)}{1-N^{\prime}\left(x_{0}\right)}+0+\frac{1}{2}\left(\frac{N\left(x_{0}\right)}{1-N^{\prime}\left(x_{0}\right)}\right)^{2} N^{\prime \prime}\left(x_{0}\right) \\
& =x_{0}+\frac{g\left(x_{0}\right)-x_{0}}{1-g^{\prime}\left(x_{0}\right)}+\frac{1}{2}\left(\frac{g\left(x_{0}\right)-x_{0}}{1-g^{\prime}\left(x_{0}\right)}\right)^{2} \frac{g^{\prime \prime}\left(x_{0}\right)}{1-g^{\prime}(\gamma)} \\
& =\frac{g\left(x_{0}\right)-x_{0} g^{\prime}\left(x_{0}\right)}{1-g^{\prime}\left(x_{0}\right)}+\frac{1}{2}\left(\frac{g\left(x_{0}\right)-x_{0}}{1-g^{\prime}\left(x_{0}\right)}\right)^{2} \frac{g^{\prime \prime}\left(x_{0}\right)}{1-g^{\prime}(\gamma)} .
\end{aligned}
$$

From this formulation, we suggest the iteration scheme as follows.

Algorithm 4 For any initial value $x_{0}$, we compute the approximation solution $x_{n+1}$, by the iterative method. Predictor step:

$$
y_{n}=\frac{g\left(x_{n}\right)-x_{n} g^{\prime}\left(x_{n}\right)}{1-g^{\prime}\left(x_{n}\right)} .
$$

Corrector step:

$$
x_{n+1}=\frac{g\left(y_{n}\right)-y_{n} g^{\prime}\left(y_{n}\right)}{1-g^{\prime}\left(y_{n}\right)}+\frac{\left(y_{n}-g\left(y_{n}\right)\right)^{2} g^{\prime \prime}\left(y_{n}\right)}{2\left(1-g^{\prime}\left(x_{n}\right)\right)\left(1-g^{\prime}\left(y_{n}\right)\right)^{2}} .
$$

\section{Convergence Analysis}

In this section, we present the convergence analysis of algorithm 3 and algorithm 4 established in this paper.

Theorem 5 Let $f: I \subset \mathbb{R} \rightarrow \mathbb{R}$ for an open interval I and consider that the nonlinear equation $f(x)=0$ 
(or $x=g(x)$ ) has simple root $\alpha \in I$, where $g(x): I \subset \mathbb{R} \rightarrow \mathbb{R}$ be sufficiently smooth in the neighborhood of the root $\alpha$. If $x_{0}$ is sufficiently close to $\alpha$ then the two-step iterative method defined by algorithm 3 has fourth order convergence.

Proof. Let

$$
G(x)=\frac{g\left(\frac{g(x)-x g^{\prime}(x)}{1-g^{\prime}(x)}\right)-\frac{g(x)-x g^{\prime}(x)}{1-g^{\prime}(x)} g^{\prime}\left(\frac{g(x)-x g^{\prime}(x)}{1-g^{\prime}(x)}\right)}{1-g^{\prime}\left(\frac{g(x)-x g^{\prime}(x)}{1-g^{\prime}(x)}\right)} .
$$

Since $\alpha$ is the root of $f(x)=0$ and $x=g(x)$ is the functional equation of $f(x)=0$, therefore $\alpha=g(\alpha)$. From (20), using Maple software, we have

$$
\begin{gathered}
G(\alpha)=\alpha \\
G^{\prime}(\alpha)=0 \\
G^{\prime \prime}(\alpha)=0 \\
G^{\prime \prime \prime}(\alpha)=0 \\
G^{i v}(\alpha)=\frac{-\left(g^{\prime \prime}(c)\right)^{3}}{\left(1-g^{\prime}(c)\right)^{3}} \neq 0 .
\end{gathered}
$$

Hence, by theorem 1, the algorithm 3 has fourth order convergence.

Theorem 6 Let $f: I \subset \mathbb{R} \rightarrow \mathbb{R}$ for an open interval I and consider that the nonlinear equation $f(x)=0$ (or $x=g(x)$ ) has simple root $\alpha \in I$, where $g(x): I \subset \mathbb{R} \rightarrow \mathbb{R}$ be sufficiently smooth in the neighborhood of the root $\alpha$. If $x_{0}$ is sufficiently close to $\alpha$ then the two-step iterative method defined by algorithm 4 has fifth order convergence.

Proof. Let

$$
\begin{aligned}
G(x)= & \frac{g\left(\frac{g(x)-x g^{\prime}(x)}{1-g^{\prime}(x)}\right)-\frac{g(x)-x g^{\prime}(x)}{1-g^{\prime}(x)} g^{\prime}\left(\frac{g(x)-x g^{\prime}(x)}{1-g^{\prime}(x)}\right)}{1-g^{\prime}\left(\frac{g(x)-x g^{\prime}(x)}{1-g^{\prime}(x)}\right)} \\
& +\frac{\left(\frac{g(x)-x g^{\prime}(x)}{1-g^{\prime}(x)}-g\left(\frac{g(x)-x g^{\prime}(x)}{1-g^{\prime}(x)}\right)\right)^{2} g^{\prime \prime}\left(\frac{g(x)-x g^{\prime}(x)}{1-g^{\prime}(x)}\right)}{2\left(1-g^{\prime}\left(\frac{g(x)-x g^{\prime}(x)}{1-g^{\prime}(x)}\right)\right)^{2}\left(1-g^{\prime}(x)\right)} .
\end{aligned}
$$

Since $\alpha$ is the root of $f(x)=0$ and $x=g(x)$ is the functional equation of $f(x)=0$, therefore $\alpha=g(\alpha)$. From (20), using Maple software, we have

$$
\begin{aligned}
& G(\alpha)=\alpha \\
& G^{\prime}(\alpha)=0 \\
& G^{\prime \prime}(\alpha)=0 \\
& G^{\prime \prime \prime}(\alpha)=0 \\
& G^{i v}(\alpha)=0
\end{aligned}
$$


Table 1. Numerical comparison.

\begin{tabular}{|c|c|c|c|c|}
\hline Examples & Functional eq. & IT & $X_{n}$ & $f\left(x_{n}\right)$ \\
\hline$f_{1}, x_{0}=-1$ & $g(x)=\sin x+\frac{1}{x+\sin x}$ & & & \\
\hline $\mathrm{CM}$ & & 5 & -1.404491648315341226350868178 & $-2.0 \mathrm{e}^{-63}$ \\
\hline NR & & 5 & -1.404491648315341226350868176 & $1.2 \mathrm{e}^{-28}$ \\
\hline $\mathrm{J}_{1}$ & & 3 & -1.404491648215341226035086891 & $1.82 \mathrm{e}^{-25}$ \\
\hline $\mathrm{J}_{2}$ & & 3 & -1.404491648215341242094290841 & $3.99 \mathrm{e}^{-17}$ \\
\hline $\mathrm{S}_{1}$ & & 3 & -1.404491648215341226035086818 & $6.11 \mathrm{e}^{-74}$ \\
\hline $\mathrm{S}_{2}$ & & 3 & -1.404491648215341226035086818 & $6.11 \mathrm{e}^{-74}$ \\
\hline$f_{2}, x_{0}=2$ & $g(x)=\frac{\mathrm{e}^{x}-2}{x-3}$ & & & \\
\hline $\mathrm{CM}$ & & 4 & 0.257530285439860760455367304 & $1.0 \mathrm{e}^{-63}$ \\
\hline NR & & 5 & 0.257530285439860760455367306 & $-6.0 \mathrm{e}^{-24}$ \\
\hline $\mathrm{J}_{1}$ & & 3 & 0.257530285439860760455367304 & $2.95 \mathrm{e}^{-55}$ \\
\hline $\mathrm{J}_{2}$ & & 3 & 0.257530285439860760455367303 & $3.90 \mathrm{e}^{-27}$ \\
\hline $\mathrm{S}_{1}$ & & 4 & 0.257530285439860760455367305 & $2.58 \mathrm{e}^{-46}$ \\
\hline $\mathrm{S}_{2}$ & & 4 & 0.257530285439860760455367305 & $2.58 \mathrm{e}^{-46}$ \\
\hline$f_{3}, x_{0}=1.7$ & $g(x)=\cos x$ & & & \\
\hline $\mathrm{CM}$ & & 4 & 0.739085133215160641655372087 & 0 \\
\hline NR & & 4 & 0.739085133215160641655372089 & $1.4 \mathrm{e}^{-15}$ \\
\hline $\mathrm{J}_{1}$ & & 3 & 0.739085133215160641655312087 & $5.45 \mathrm{e}^{-65}$ \\
\hline $\mathrm{J}_{2}$ & & 3 & 0.739085133215160641655312087 & $3.75 \mathrm{e}^{-55}$ \\
\hline $\mathrm{S}_{1}$ & & 3 & 0.739085133215160641655312087 & $5.45 \mathrm{e}^{-65}$ \\
\hline $\mathrm{S}_{2}$ & & 3 & 0.739085133215160641655312087 & $5.45 \mathrm{e}^{-65}$ \\
\hline$f_{4}, x_{0}=3.5$ & $g(x)=1+\sqrt{\frac{1}{x-1}}$ & & & \\
\hline $\mathrm{CM}$ & & 5 & 2 & 0 \\
\hline NR & & 5 & 2.000000000000000000000000008 & $1.03 \mathrm{e}^{-23}$ \\
\hline $\mathrm{J}_{1}$ & & 4 & 2.000000000000000000000000000 & $2.05 \mathrm{e}^{-42}$ \\
\hline $\mathrm{J}_{2}$ & & 4 & 2.000000000000000000000000000 & $4.21 \mathrm{e}^{-52}$ \\
\hline $\mathrm{S}_{1}$ & & 3 & 2.000000000000000000000000000 & $3.31 \mathrm{e}^{-43}$ \\
\hline $\mathrm{S}_{2}$ & & 3 & 2.000000000000000000000000000 & $3.31 \mathrm{e}^{-43}$ \\
\hline \multicolumn{5}{|l|}{$f_{5}, x_{0}=1.5$} \\
\hline $\mathrm{CM}$ & & 5 & 2.154434690031883721759235667 & $-5.00 \mathrm{e}^{-63}$ \\
\hline NR & & 5 & 2.154434690031883721759235663 & $3.10 \mathrm{e}^{-42}$ \\
\hline $\mathrm{J}_{1}$ & & 3 & 2.154434690031883721759293567 & $6.85 \mathrm{e}^{-27}$ \\
\hline $\mathrm{J}_{2}$ & & 3 & 2.154434690031883721759293566 & $3.19 \mathrm{e}^{-27}$ \\
\hline $\mathrm{S}_{1}$ & & 3 & 2.1544346900318837217592935665 & $1.08 \mathrm{e}^{-64}$ \\
\hline $\mathrm{S}_{2}$ & & 3 & 2.1544346900318837217592935665 & $1.08 \mathrm{e}^{-64}$ \\
\hline$f_{6}, x_{0}=3.5$ & $g(x)=\frac{1}{7}\left(30-x^{2}\right)$ & & & \\
\hline $\mathrm{CM}$ & & 8 & 3.0000000000000000000000000003 & $2.00 \mathrm{e}^{-62}$ \\
\hline NR & & 7 & 3.0000000000000000000000000006 & $-2.1 \mathrm{e}^{-29}$ \\
\hline $\mathrm{J}_{1}$ & & 7 & 3.0000000000000000000000000000 & $1.16 \mathrm{e}^{-94}$ \\
\hline $\mathrm{J}_{2}$ & & 6 & 3.0000000000000000000000000377 & $4.90 \mathrm{e}^{-25}$ \\
\hline $\mathrm{S}_{1}$ & & 3 & 3.000000000000000000000000000 & $4.17 \mathrm{e}^{-90}$ \\
\hline $\mathrm{S}_{2}$ & & 3 & 3.0000000000000000000001629758 & $2.12 \mathrm{e}^{-21}$ \\
\hline
\end{tabular}




$$
G^{v}(\alpha)=\frac{15(g(c))^{4}}{\left(1-g^{\prime}(c)\right)^{4}} \neq 0
$$

Hence, by theorem 1, the algorithm 4 has fifth order convergence.

\section{Numerical Tests}

In this section, we shall solve some nonlinear equations to illustrate the efficiency of the newly developed fourth and fifth order iterative methods by using algorithm $3\left(\mathrm{~S}_{1}\right)$ and algorithm $4\left(\mathrm{~S}_{2}\right)$ in this paper. We shall make comparison with four and fifth order iterative methods established earlier such as the method of Chun (CM) [3], the method of Noor (NR) [4], the algorithm $2.1\left(\mathrm{~J}_{1}\right)$ and algorithm $2.2\left(\mathrm{~J}_{2}\right)$ of Javidi [8]. We use $\in=10^{-15}$. The following criterias are used for computer programs:

$$
\begin{aligned}
& \text { (i) } \quad\left|x_{n}-x_{n-1}\right|<\epsilon \\
& \text { (ii) } \quad\left|f\left(x_{n}\right)\right|<\epsilon .
\end{aligned}
$$

The examples are same as in Chun [3] and Noor [4].

$$
\begin{gathered}
f_{1}(x)=\sin ^{2} x-x^{2}+1 \\
f_{2}(x)=x^{2}-\mathrm{e}^{x}-3 x+2 \\
f_{3}(x)=\cos x-x \\
f_{4}(x)=(x-1)^{3}-1 \\
f_{5}(x)=x^{3}-10 \\
f_{6}(x)=\mathrm{e}^{x^{2}+7 x-30}-1
\end{gathered}
$$

\section{Conclusion}

In this paper, we have developed two new iterative methods of order four and five for the solution of nonlinear equations based on homotopy perturbation method. To derive these iteration schemes, we have used a very simple technique. Convergence analysis is also discussed. To check convergence, performance and validity, we have applied these iterative methods to solve some nonlinear equations. From Table 1, we see the validity and efficiency of these iterative methods as compared with other methods. Thus our newly established iterative methods are interesting and reliable alternative methods of existing methods in literature of order four and order five for solving nonlinear equations under consideration. Also our methods converge faster than existing methods of order four and five such as Noor [4] and Javidi [8].

\section{References}

[1] Abbasbandy, S. (2003) Improving Newton-Raphson Method for Nonlinear Equations by Modified Adomain Decomposition Method. Applied Mathematics and Computation, 145, 887-893. http://dx.doi.org/10.1016/S0096-3003(03)00282-0

[2] Adomain, G. (1989) Nonlinear Atochastic Systems and Applications to Physics. Kluwer Academy Publishers, Dordrecht. http://dx.doi.org/10.1007/978-94-009-2569-4

[3] Chun, C. (2005) Iterative Methods Improving Newton's Method by Decomposition Method. Applied Mathematics and Computation, 50, 1559-1568. http://dx.doi.org/10.1016/j.camwa.2005.08.022

[4] Noor, M.A. and Inayat, K. (2006) Three-Step Iterative Methods for Nonlinear Equations. Applied Mathematics and Computation, 183, 322-327. http://dx.doi.org/10.1016/j.amc.2006.05.055

[5] Noor, M.A., Inayat, K. and Tauseef, M.D. (2006) An Iterative Method with Cubic Convergence for Nonlinear Equations. Applied Mathematics and Computation, 183, 1249-1255. http://dx.doi.org/10.1016/j.amc.2006.05.133

[6] Babolian, E. and Biazar, J. (2002) On the Order of Convergence of Adomain Method. Applied Mathematics and Com- 
putation, 130, 383-387. http://dx.doi.org/10.1016/S0096-3003(01)00103-5

[7] Daftardar-Gejji, V. and Jafari, H. (2006) An Iterative Method for Solving Nonlinear Functional Equations. Journal of Mathematical Analysis and Applications, 316, 753-763. http://dx.doi.org/10.1016/j.jmaa.2005.05.009

[8] Javidi, M. (2009) Fourth Order and Fifth Order Iterative Methods for Nonlinear Algebraic Equations. Mathematical and Computer Modelling, 50, 66-71.

[9] Kang, S.M., et al. (2013) A New Second Order Iteration Method for Solving Nonlinear Equations. Handawi Publishing Company, Abstract and Applied Analysis, 2013, Article ID: 48706.

[10] He, J.H. (1999) Homotopy Perturbation Technique. Computer Methods in Applied Mechanics and Engineering, 178, 257-262. http://dx.doi.org/10.1016/S0045-7825(99)00018-3 